\title{
Освоение EUV-литографии в серийном производстве: перспективы и проблемы
}

\author{
М. Макушин ${ }^{1}$, В. Мартынов, д. т.н. ${ }^{2}$
}

УДК 621.37| ВАК 05.27 .06

\begin{abstract}
Появление коммерчески жизнеспособной литографии на источниках излучения в предельной УФ-области спектра (EUV, длина волны 13,5 нм) ожидалось в 2005 году на 90-нм топологиях. После более 20 лет работ и миллиардов затраченных долларов EUV-литография начала использоваться в массово-поточном производстве. Первопроходцем в июне 2018 года стала корпорация Samsung, позже к ней присоединился крупнейший кремниевый завод TSMC. В текущем году ожидается поставка 30 EUV-установок корпорации ASML. Тем не менее, по мере освоения в серийном производстве данной технологии обнаруживаются новые проблемы практически по всем ее аспектам.
\end{abstract}

EUV-литография окончательно вошла в серийное производство у ряда крупных производителей на уровне топологий порядка 10 /7 нм. Она предлагает повышенную разрешающую способность, а также более простой технологический процесс и правила проектирования с меньшим числом ограничений. Кроме того, для приборов с топологическими нормами менее 7 нм EUV-литография обеспечивает преимущество в издержках по сравнениюс другими методами литографии. Сама идея EUV-литографии возникла как путь ухода от методик многократного формирования рисунка в рамках иммерсионной 193-нм литографии и формирования структур «за один прогон".

Однако уже сейчас начинает возникать вопрос - а не понадобится ли на 5-нм топологиях двукратное и даже многократное EUV-формирование рисунка? Не исчезнет ли в связи с этим простота технологического процесса и преимущество по издержкам?

В любом случае EUV-литография окажет значительное воздействие на полупроводниковую промышленность. Хотя производителям ИС удалось продлить использование традиционной 193-нм (иммерсионной) литографии и методик многократного формирования рисунка на технологический уровень $10 / 7$ нм, выйти за пределы этого уровня по наиболее сложным топологическим элементам без использования EUV-литографии становится слишком сложно и дорого. Проектные нормы 7 нм являются точкой перехода, на которой еще ведутся споры о том, какой подход использовать дальше. При этом даже если EUV и применяется для формирования элементов с критическими размерами, другие части данного прибора вполне могут быть созданы с использованием традиционных методик формирования рисунка [1]

ЦНИи «Электроника», главный специалист, mmackushin@gmail.com. ФГБНУ “Аналитический центр», проф., Минобрнауки РФ, эксперт.

\section{ПРОБЛЕМЫ ОСВОЕНИЯ ЕUV-ЛИТОГРАФИИ В СЕРИЙНОМ ПРОИЗВОДСТВЕ ИС}

EUV-литография находится в стадии становления. Время простоя оборудования, а также уровни дефектности пока остаются достаточно высокими. Как и предыдущие поколения литографии, EUV-литография сталкивается с компромиссами между разрешающей способностью, неравномерностью краев линий (LER) и экспозиционной дозой. По сравнению с лучшими вариантами экспонирования в рамках 193-нм литографии, EUV-литография предлагает увеличение разрешающей способности в 2,5 раза при увеличении плотности пикселов в шесть раз. В течение нескольких поколений технологий литографии величина LER оставалась постоянной, в то время как общие размеры топологических элементов уменьшались. По всей видимости, LER становится ограничивающим фактором для улучшения разрешающей способности в целом

LER тесно связана с изменчивостью параметров процесса. Современные EUV-источники излучения генерируют в 14 раз меньше фотонов, чем их 193-нм аналоги. Случайное распределение этих фотонов (т.н. дробовой шум) может привести к тому, что некоторые области пластины окажутся недоэкспонированными. При этом, с одной стороны, увеличение экспозиционной дозы может привести к увеличению производительности литографической установки и снижению уровня дефектности, связанного с недоэкспонированием, но оно же, с другой стороны, может увеличить уровень дефектности, связанный с переэкспонированием.

\section{Увеличение яркости источника излучения и оптики}

слабым местом EUV-литографии всегда была недостаточная мощность источника излучения. В то время как излучение установок 193-нм литографии поступает непосредственно от ArF-лазеров, то EUV-фотоны испускаются 
плазмой, созданной перегретыми каплями олова. При использовании для генерации подобной плазмы лазера мощностью 20 кВт концентраторная оптика захватывает не более 250 Вт 13,5-нм излучения. Многослойные Mo/ Si-зеркала поглощают часть этой мощности, и на резист попадает значительно меньше 250 Вт. Правда, уже доступны 250-Вт источники EUV-излучения, а при кратковременных импульсах достигается мощность 450 Вт. До конца 2019 года на рынке могут появиться 330-Вт источники.

Разработчики систем экспонирования стремятся более эффективно использовать доступные фотоны. В оптике с числовой апертурой 0,55 можно решить проблему эффективности за счет использования анаморфных объективов с 8/4-кратным увеличением, применяемым только в направлении сканирования. Это наполовину сокращает размер поля экспонирования, но не решает полностью проблемы производительности EUV-установки. В случае экспонирования сначала первого, а потом второго полуполя всех пластин одной партии можно снизить накладные расходы на перемещение пластины и промежуточного шаблона [2].

\section{EUV-шаблоны}

EUV-шаблоны существенно отличаются от оптических шаблонов - это многослойные структуры, состоящие из чередующихся слоев кремния и молибдена поверх подложки, в результате чего их толщина достигает 250-350 нм. Далее, EUV-излучение хорошо поглощается многими материалами, поэтому требуются отражающие шаблоны - необработанные шаблоны с несколькими слоями. Ихпоглощающий слой, с одной стороны, должен быть максимально тонким, а с другой - обеспечивать хороший контраст отражающей части шаблона.

Отражающий EUV-шаблон находится в вакууме - это еще одно отличие EUV-литографии от предшествующих процессов оптической литографии. Для реализации EUV-шаблонов требуются пелликулы, но идеального решения для массового производства ИС по данному вопросу пока нет - опятьтаки из-за сильного поглощения EUV-излучения практически всеми материалами. При этом необходимо обеспечить баланс всех различных пленок шаблона по различным параметрам, включая электростатику. У EUV-шаблонов возникает проблема дефектности обратной стороны - в рамках 193-нм литографии такой проблемы не существовало. Обеспечить плоскостность отражающего шаблона намного сложнее. При увеличении мощности облучения обостряется проблема нагрева и EUV-шаблонам требуется материал с низким тепловым расширением. В итогеполучается, что стоимость необработанного EUV-шаблона значительно больше цены аналогичных структур предшествующих поколений литографии

Существует ряд вопросов относительно собственно EUV-шаблонов и ожиданий разработчиков. На раннем этапе освоения EUV-литографии не требуется использование сложных методик коррекции эффекта оптической близости или технологии инверсионной литографии. Это облегчает проблему объема данных, требования к минимальным размерам топологических элементов на шаблоне и операции контроля/проверки. На данный момент в большинстве EUV-шаблонов не используется методикa SRAF", популярная в рамках традиционной "глубокой» УФ-литографии (DUV, эксимерные лазеры 248-193 нм для топологий от 90 до 45 / 20 нм) и технологиях многократного формирования рисунка. Это позволяет использовать в EUV-литографии более простые виды рисунков. По мере дальнейшего освоения и оптимизации EUV-литографии возможно наращивание сложности процесса, связанное с необходимостью получения максимальной резкости изображения, уменьшения стохастических эффектов и т. п. Очевидно, что в дальнейшем EUV-литография потребует более активного использования методов вычислительной литографии [3].

EUV-шаблоны можно формировать с существующей инфраструктурой. Многообещающей является готовность к использованию электронно-лучевыхустановок непосредственного формирования рисунка шаблона при помощи множественных лучей (multi-beam e-beam mask writers).

\section{Резисты, дефекты и формирование рисунка}

EUV-резисты имеют ряд особенностей, обусловленных относительно низким числом доступных фотонов. Они (резисты) должны быть достаточно чувствительными для формирования изображения, но при этом структуры пластин с высоким аспектным отношением требуют бо́льшего времени травления и лучшей стойкости резистов ктравлению. Предпочтительны тонкие слои резиста, так как высокие, узкие столбцы резиста (получаемые при травлении толстых слоев) имеют тенденцию к обрушению. Недостатком же тонких слоев резиста является захват меньшего числа фотонов и меньшая устойчивость к травлению. Поэтому полупроводниковая промышленность ищет альтернативы резистам (химически усиленным), используемым в существующих процессах литографии.

\footnotetext{
SRAF (sub-resolution assist features) - вспомогательные топологические элементы с размерами, меньшими, чем разрешающая способность литографии, используемые для расширения технологического окна процесса и "тонкой настройки" размеров и конфигурации элементов топологии. Имеется тенденция совместного использования с методикой коррекции оптической близости (optical proximity correction, OPC)

*: Computational lithography (computational scaling) - вычислительная литография, набор математическихи алгоритмических подходов для улучшения разрешающей способности фотолитографии. Применяется с 2008 года в связи с переходом к процессам с топологиями 22 нм и менее.
} 
Наиболее изученной альтернативой химически усиленному резисту является материал, состоящий из кластеров оксида металла, окруженных органической оболочкой. В подобном материале неорганическое ядро обеспечивает поглощение EUV-излучения и сопротивление травлению, а органическая оболочка определяет технологичность пленки. Отмечается, что органическая оболочка фактически растворяется в проявителе или вступает в перекрестную связь с соседними молекулами. Небольшие изменения органической оболочки помогли улучшить эксплуатационные характеристики резистов на основе кластеров оксида гафния и циркония.

\section{Управление дефектами технологичности}

Одним из показателей технологичности процесса, связанного с обработкой резиста, является наличие бездефектного окна процесса" - независимо от использования различных химических веществ и соединений. По мере уменьшения промежутков между линиями и увеличения LER между соседними линиями могут образовываться микромостики. Если они сохраняются в процессе травления, то приводят к коротким замыканиям. Наименьшее расстояние, при котором число микромостиков остается ниже приемлемого порога дефектности, является минимальным технологическим размером.

При другой крайности - расширении промежутков между линиями и уменьшении ширины линий - могут появляться разрывы линий. В готовых приборах они становятся разомкнутыми цепями. Эта сторона бездефектного окна процесса определяется минимальной шириной линии, при которой число разрывов остается ниже приемлемого порога дефектности. В матрице контактных отверстий бездефектное окно процесса находится между наименьшими контактными отверстиями, которые могут оставаться открытыми, и наибольшими отверстиями, которые можно сформировать без слияния с соседними отверстиями. Технологичный процесс обычно имеет области "стохастических отказов" по обе стороны

* Process window - окно процесса, набор значений параметров процесса, позволяющий изготавливать ИС, и работающий при желаемых спецификациях. Например, окно процесса литографии обычно определяется как набор пунктов (фокус, экспозиция и т.д.) для контроля разбросов критических размеров в пределах $10 \%$ бездефектного окна процесса, за которыми число наблюдаемых отказов резко падает (рис. 1).

Вышедшие из строя межслойные переходы, даже на уровне одного случая на миллиард, могут снизить выход годных на 25\% (максимум). В хорошо отлаженном процессе почти все размеры топологических элементов попадают в плотное распределение вокруг среднего значения критических размеров.

\section{Пострезистная обработка и перенос рисунка}

Формируемые на пластине топологические элементы являются результатом взаимодействия установки экспонирования с резистом, а также таких факторов, как профиль травления, воспроизводимость осаждения и напряженность пластины. В целом, легче изменить резисты и процессы переноса рисунка, чем установку экспонирования. Также проще ввести в процесс монослой, полученный методом самосборки, или улучшить удаление побочных продуктов после травления, чем увеличить полезный выход лазерно-индуцированной плазмы. Часто этапы обработки после литографии могут исправить дефекты резиста, внесенные экспонированием.

Материалы, получаемые методами самосборки, впервые рассматривались как одно из средств улучшения разрешающей способности 193-нм литографии. Методика непосредственной самосборки (directed self-assembly, DSA) остается актуальной и в эпоху EUV-литографии. DSA-монослои улучшают перенос рисунка и снижают ошибки установки угла кристалла в заданное положение (edge placement error, EPE) при выполнении операций, следующих за этапом литографии [2]. 


\section{АКЦИОНЕРНОЕ ОБЩЕСТВО «ЗАВОД ПОЛУПРОВОДНИКОВЫХ ПРИБОРОВ»}

\section{КОНТАКТНЫЕ УСТРОЙСТВА ЭКБ ДЛЯ ОТЕЧЕСТВЕННОГО И ИМПОРТНОГО ПРОИЗВОДСТВА}

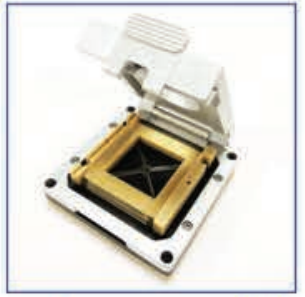

Модульные КУ без механизма закрывания

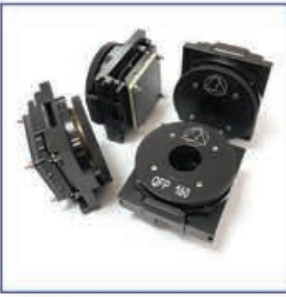

Модульные КУ с механизмом закрывания

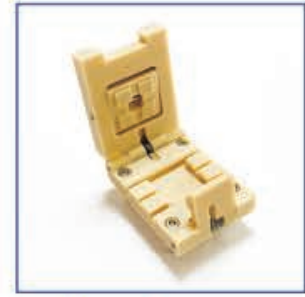

КУ для безвыводных корпусов (микросхем)

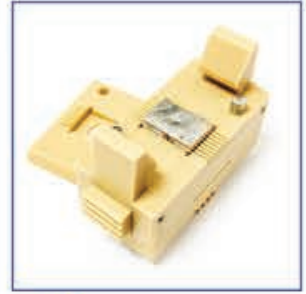

КУ для силовых корпусов (микросхем)

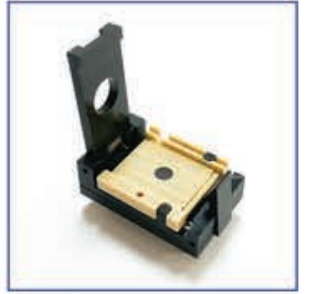

КУ со спутникомносителем

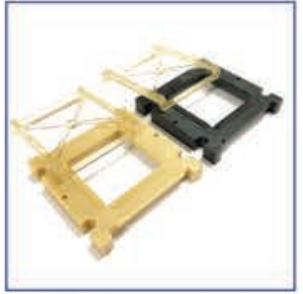

Спутникиносители

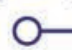

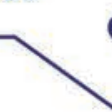

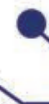




\section{ВОПРОСЫ ОДНОКРАТНОГО И МНОГОКРАТНОГО EUV-ФОРМИРОВАНИЯ РИСУНКА}

Несмотря на существующие проблемы, кремниевые заводы готовятся к промышленному освоению EUV-технологии. Например, крупнейший «чистый» кремниевый завод TSMC для производства первого поколения 7-нм ИС использует 193-нм иммерсионную литографию и методику многократного формирования рисунка. В этом году корпорация начнет применять EUV-литографию при производстве второго поколения 7-нм ИС, по крайней мере, для формирования некоторых критическихслоев. В следующем году корпорация планирует начать выпуск 5-нм ИС, причем число слоев, для формирования которых применяется EUV-литография, будет существенно увеличено. Корпорация Samsung намерена использовать EUV-литографию для изготовления 7-нм ИС, а позднее и 5-нм ИС. Корпорация Intel планирует использовать EUV-литографию на уровне 7-нм процессов, которые, по утверждениям ее специалистов, будут аналогичны по основным параметрам 5-нм процессам Samsung и TSMC.

На уровне 7-нм технологического процесса EUV-литография, в частности, пригодна для однократного формирования рисунков топологических элементов с шагом 38 или 36 нм. Данный подход также может использоваться для формирования контактов/межслойных переходов, которые обладают бо́льшим шагом. Но здесь начинаются компромиссы. Для формирования металлических слоев в рамках7-нм процесса подходит 193-нм иммерсионная литография и методика многократного формирования рисунка, использование которых удалось продлить до этого уровня. Но 7-нм проектные нормы также являются отправной точкой использования технологии однократного EUV-формирования рисунка. Это означает, что производителям ИС придется решать, какую из этих технологий применять для производства своих ИС.

Кроме того, поскольку EUV-литография сильно задержалась с освоением в производстве (первоначально предполагалось начать ее использование в 2005 году на 90-нм технологическом уровне), окно существования однократного EUV-формирования рисунка сузилось до 7-нм процесса. Действительно, при том что стартовая точка для нее это шаг элементов порядка 38/36 нм, предел возможностей данной методики достигается уже при шаге около 32/30 нм. Шаг менее 30 нм потребует использования технологии двойного EUV-формирования рисунка, применение которой намечено на уровне 5-нм процессов (ввиду того, что на уровне 7-нм процесса методика двойного формирования рисунка не практична).

Заказчики кремниевых заводов могут запустить конструкции своих 7-нм ИС в производство с использованием методики однократного EUV-формирования рисунка и дойти (достаточно быстро) до пределов ее возможностей. Но при этом они должны решить, стоит ли им тратить средства на процесс с ограниченными технологическими (возможно и временными) границами существования

Правда, существует возможность использования однократного EUV-формирования рисунка на меньших размерахтопологических элементов. Использование данной методики в основном ориентировано на достаточно гибкие конструкции. Достичь снижения шага элементов до 32 нм вполне возможно, но перейти к меньшим топологическим размерам с существующими резистами - очень проблематично. Также встает вопрос создания робастного EUV-peзиста с необходимой чувствительностью и точной выдержкой дозы облучения.

Доза облучения играет важную роль в процессе EUV-литографии. По мнению отраслевых экспертов, производительность EUV-сканера тесно связана с мощностью источника излучения. Например, при дозе 30 мДж/ см² EUV-cканер с источником излучения мощностью 250 Вт способен обрабатывать 104-105 пластин в час (данные корпорации ASML). При работе c EUV-литографией производители ИС для обработки резиста используют различные дозы, при этом 20/30 мДж/см² считается низкой дозой, а 80 мДж/см² высокой. В обоих случаях существуют определенные проблемы. Предположим, есть шаблон с плотным шагом. Это требует очень большой дозы облучения, в результате чего производительность установки снижается. Однако при использовании больших доз уменьшается дефектность. Так, при дозе 30 мДж/ см² рисунки с плотным шагом элементов, полученные методом однократного EUV-формирования, демонстрируют больший уровень дефектности, чем те же самые рисунки, сформированные при использовании дозы 80 мДж/ $\mathrm{CM}^{2}$

В случае применения резистов лучшего качества при использовании однократного EUV-формирования рисунка можно создать топологические элементы с плотным шагом практически без дефектов, но резисты этого типа по-прежнему находятся в стадии НИОКР.

Некоторые отраслевые специалисты считают, что методика однократного формирования рисунка имеет больше смысла, чем ее многократная альтернатива. Действительно, лучшеудвоить дозу облучения, чем заниматься двойным формированием рисунка. При этом важным аспектом является точность совмещения шаблона. Для ее увеличения и/или избежания неточного совмещения шаблонов еще в рамках 193-нм иммерсионной литографии сприменением методик двукратного/многократного формирования рисунка был реализован “спейсерный» подход. Его суть - использование вспомогательных топологических элементов (размеры которых могут быть меньше разрешающей способности используемой технологии литографии) - спейсеров (spacers), позволяющихповысить точность размещения основных, в первую очередь критических, топологических элементов, а также повысить точность совмещения шаблона с обрабатываемой пластиной (рис. 2). Этот подход также 
хорошо вписывается в EUV-литографию-какдля однократного, так и для многократного формирования рисунка.

Методика многократного формирования рисунка, как и методика однократного формирования, обладает своим набором трудностей. Если клиенты кремниевого завода переходят к 5-нм технологическому процессу, им потребуется осуществлять двойное формирование многих топологических элементов. Специалисты корпорации ASML подчеркивают, что они всегда планировали переход от однократного EUV-формирования рисунка к многократному - по аналогии с иммерсионной литографией и ее аналогичными вариациями. Соответственно, заранее было известно, что двойное EUV-формирование рисунка будет дороже однократного. Кроме того, двойное EUV-формирование рисунка на уровне 5-нм технологий намного более выгодная альтернатива 193-нм литографии с методиками многократного формирования рисунка. Дело не только в том, что двойной цикл EUV короче и дешевле (в массовом производстве при переходе за определенный уровень объемов выпуска), но и надежнее. Для многократного 193-нм иммерсионного формирования рисунка потребуется от 6 до 8 иммерсионных этапов экспонирования. Многие специалисты полагают, что такой подход просто не будет работать.

Методика двойного EUV-формирования рисунка предполагает разделение принципиального шаблона кристалла ИС на два более простых. Каждый из них отвечает за формирование отдельного слоя. В условиях завода по обработке пластин этот метод будет обладать такими же основными этапами технологического процесса, как иммерсия/двойное формирование рисунка. В основе двойного EUV-формирования рисунка лежит идея увеличения плотности шага топологических элементов на каждом уровне (но последующее впечатывание в промежутки другой половины рисунка приведет к уменьшению шага) и использования более высокой дозы облучения. Это подразумевает применение более дорогостоящих этапов (но меньшего числа, чем при 193-нм иммерсии), но способствует уменьшению уровня дефектности

По данным корпораций IC Knowledge и TEL, отправной точкой двойного EUV-формирования рисунка является шаг топологических элементов в 26 нм. При этом для формирования контактов и межслойных переходов, которые обычно крупнее других элементов, на уровне 5-нм технологий будет использоваться методика однократного EUV-формирования рисунка.

Одним из основных направлений использования EUV в производстве будет формирование структур с очень плотным шагом элементов за счет того, что на каждом из этапов двойного формирования рисунка будет применяться шаблон с увеличенным шагом, но на втором этапе рисунок будет впечатываться в промежутки рисунка, сформированного на первом этапе. Как отмечают специалисты Межуниверситетского центра микроэлектроники (IMEC, Лёвен, Бельгия), единственный способ получения бездефектного изображения - формирование топологических элементов большего, чем нужно, размера. Подобный подход действует до шага элементов порядка 40 нм. Если же нужно получить меньший шаг (например, 38 нм), то проектировщик выходит на предел того, чего можно добиться в рамках 193-нм иммерсионной литографии при использовании методики двойного формирования рисунка с самосовмещением (self-aligned double pattering, SADP). Если же удастся в рамках однократного EUV-формирования рисунка сделать то, что ранее достигалось при использовании 193-нм иммерсии с тройным формированием рисунка, то EUV-решения становятся не просто рентабельными, а более предпочтительными, чем любые иммерсионные решения. Далее, если использовать двойное EUV-формирование рисунка, то добиться нулевой дефектности можно при низкой дозе облучения. Например, необходимо сформировать структуру с шагом элементов в 32 нм, используя двойное EUV-формирование рисунка. Для этого сначала формируется один набор топологических элементов (первый шаблон) с шагом 64 нм, затем в промежутки впечатывается второй набор топологических элементов с тем же шагом (рис. 3). В результате шаг между элементами конечного рисунка составит 32 нм, при этом дефектность будет практически нулевая, а доза излучения (на каждом из этапов) составит 30 мДж/см². В принципе, и при однократном EUV-формировании рисунка с дозой 60 мДж/см², и при двукратном EUV-формировании с дозой 30 мДж/см² задействовано практически одинаковое число фотонов, но во втором случае это осуществляется за два захода в "расслабленном" режиме.

Специалисты корпорации Applied Materials отмечают, что на первом и втором поколении 7-нм технологий все EUV-решения будут представлены подходом однократного

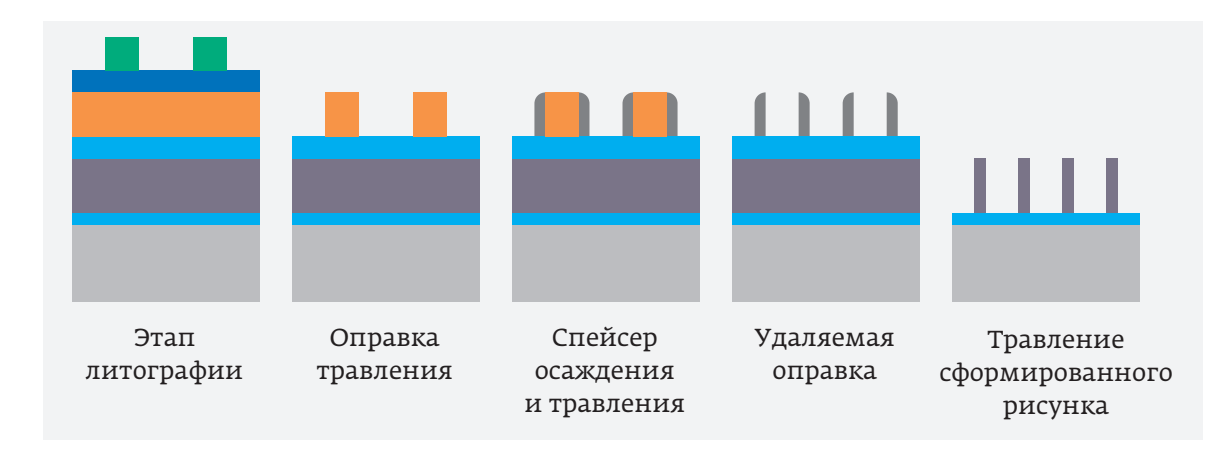

Рис. 2. Самосовмешающиеся спейсеры позволяют избежать неточного совмещения шаблона. Источник: Lam Research 


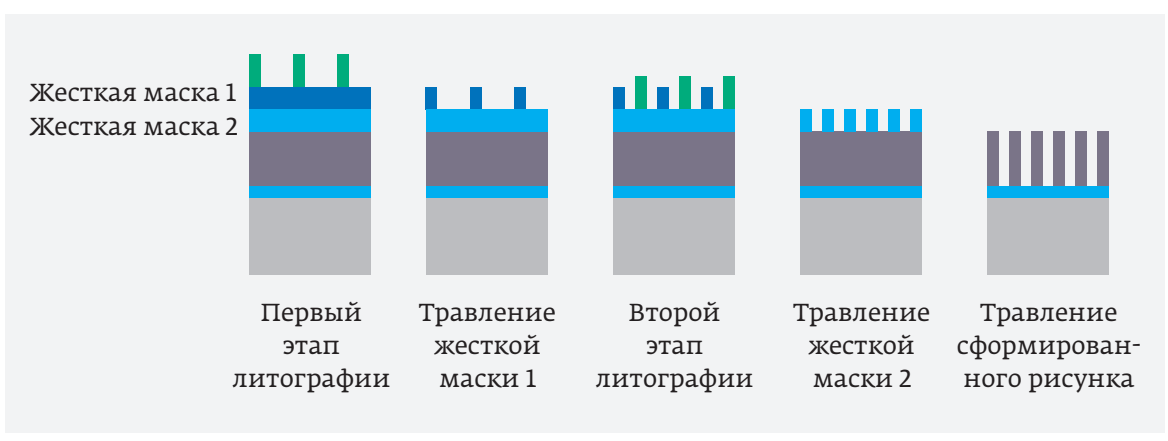

Рис. 3. Двойное формирование рисунка позволяет увеличить плотность расположения элементов. Источник: Lam Research

формирования рисунка. При переходе к меньшим проектным нормам появятся как минимум три активныхслоя металлизации, требующихся для создания двукратного формирования рисунка. Но это не означает, что подход однократного формирования рисунка становится не важным. Например, конструкция требует от самого нижнего слоя металлизации минимального шага топологических элементов, что осуществляется при помощи двукратного формирования рисунка. Но тогда вышележащие слои могут быть сделаны при помощи однократного формирования рисунка, что обеспечит значительную экономию времени и средств. При этом все операции могут выполняться с шагом 32 или 34 нм. Подобный подход будет важен на уровне проектных норм порядка 5 нм, а также 3 нм. Реализация нескольких слоев методиками многократного формирования рисунка, а остальных - методом однократного формирования, позволяет не только удешевить процесс, но и повысить производительность производственного оборудования.

Несмотря на то, что у метода многократного EUV-формирования рисунка существуют как достоинства, так и недостатки, к данному подходу существует значительный интерес. Этот интерес обусловлен не только возможностью поэтапного формирования структур с высокой плотностью шага, но и большей управляемостью стохастических проблем. Ввиду того, что иммерсионная технология применялась достаточно длительное время, полупроводниковая промышленность привыкла к методикам многократного формирования рисунка. Такой подход не является чем-то новым, многие поставщики ИС привыкли к нему, а значительная часть их конструкций совместима с данным подходом.

Однако принцип многократности формирования неразрывно связан с увеличением как издержек, так и изменчивости параметров процесса. В условиях заводов по обработке пластин требуется больше элементов контроля и управления. Любое отклонение показателей толщины пленки будет рассматриваться как изменение параметров процесса в целом. Кроме того, с ростом числа формируемых пленок увеличивается длительность цикла создания ИС. Существуют и другие проблемы. Так, например, EUV-литография не решает проблемы ошибки установки кристалла в заданное положение (edge placement error, ЕРЕ). Существуют две проблемы. По мере масштабирования допуски на размещение (элементов) становятся все меньше и меньше, поэтому возрастает потребность в методиках и схемах самосовмещения. Другой большой проблемой EUV-литографии являются стохастические явления, такие как стохастические отказы, стохастическая неравномерность краев линий [резиста] (line-edge roughness, LER), стохастика окна процесса (формирование микромостиков, разрывов линий) $[4,5]$.

Специалисты корпорации Lam Research отмечают еще один фактор, связанный с методикой однократного EUV-формирования рисунка, - малое окно использования шаблонов под однократное формирование с числовой апертурой 0,33. При получении шага менее 30 нм придется прибегать к методикам двойного формирования рисунка и, следовательно, к разложению единого шаблона на две части. Все это также способствует удорожанию стоимости EUV-литографии.

\section{ВАРИАНТЫ МНОГОКРАТНОГО ФОРМИРОВАНИЯ РИСУНКА}

Существует несколько способов реализации многократного EUV-формирования рисунка, каждый из которыхпо-своему усложняет ситуацию. Среди них:

- двойное EUV-формирование рисунка, требующеедвух этапов литографии и двух этапов травления;

- двойное EUV-формирование рисунка с самосовмещением, требующее одного этапа литографии, а также по одному этапу осаждения и травления;

- гибридный подход;

- EUV-литография, осуществляемая совместно с реализацией методики непосредственной самосборки (directed self-assembly, DSA), использующей блок-сополимеры, способные самостоятельно совмещаться и создавать рисунки.

Каждый из этих вариантов обладает своими достоинствами. Если используется EUV-формирование рисунков с помощью спейсеров, ограничивающих применение EUV-литографии, то ситуация вполне приемлема. Но если для формирования слоя требуется двойное или тройное EUV-экспонирование, процесс становится очень дорогим. Специалисты корпорации Mentor Graphics отмечают, что есть другие точки зрения на проблемы. Учитывая, что слои металлизации на данных технологических уровнях фактически являются однонаправленными дорожками, 


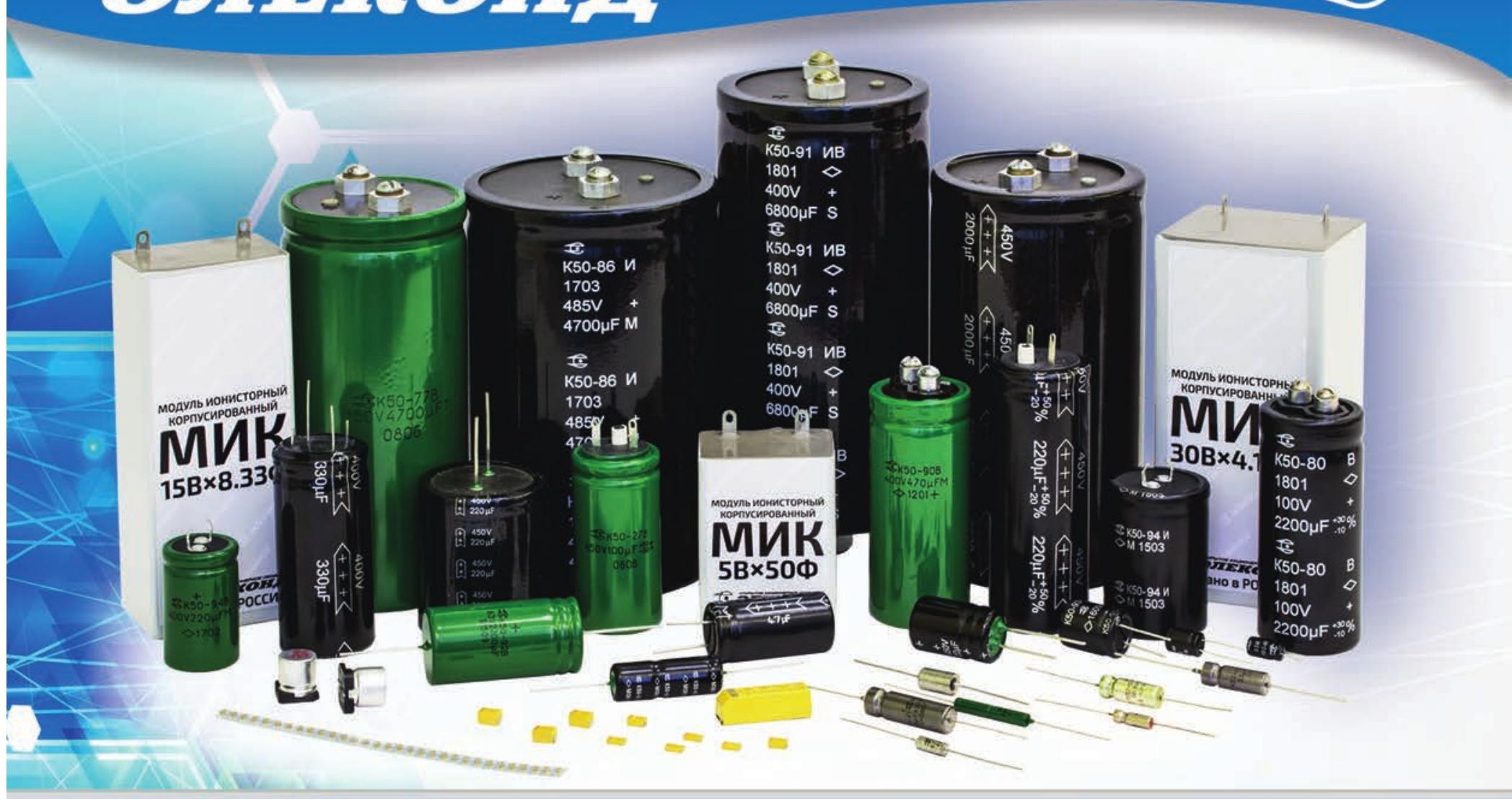

\section{Разработка и производство конденсаторов}

оксидно-электролитические алюминиевые конденсаторы К50-15, К50-17, К50-27, К50-37, К50-68, К50-74, К50-76, К50-77, К50-80, K50-81, К50-83, К50-84, К50-85, К50-86, К50-87, К50-88, К50-89, К50-90, К50-91, К50-92, К50-93, К50-94, К50-95(чип), К50-96, К50-98

объемно-пористые танталовые конденсаторы К52-1, К52-1М, К52-1БМ, К52-1Б, К52-9, К52-11, К52-17, К52-18, К52-19, К52-20, К52-21, К52-24, К52-26(чип), К52-27(чип)

оксидно-полупроводниковые танталовые конденсаторы

К53-1А, К53-7, К53-65(чип), К53-66, К53-68(чип), К53-71(чип), К53-72(чип), К53-74(чип), К53-77(чип), К53-78(чип)

суперконденсаторы (ионисторы) K58-26

накопители электрической энергии на основе модульной сборки суперконденсаторов

Система менеджмента качества сертифицирована на соответствие требованиям ISO 9001

Россия, 427968, Удмуртская Республика, г. Сарапул, ул. Калинина, 3 Тел.: (34147) 2-99-53, 2-99-89, 2-99-77, факс: (34147) 4-32-48, 4-27-53 e-mail: elecond-market@elcudm.ru, http://www.elecond.ru 


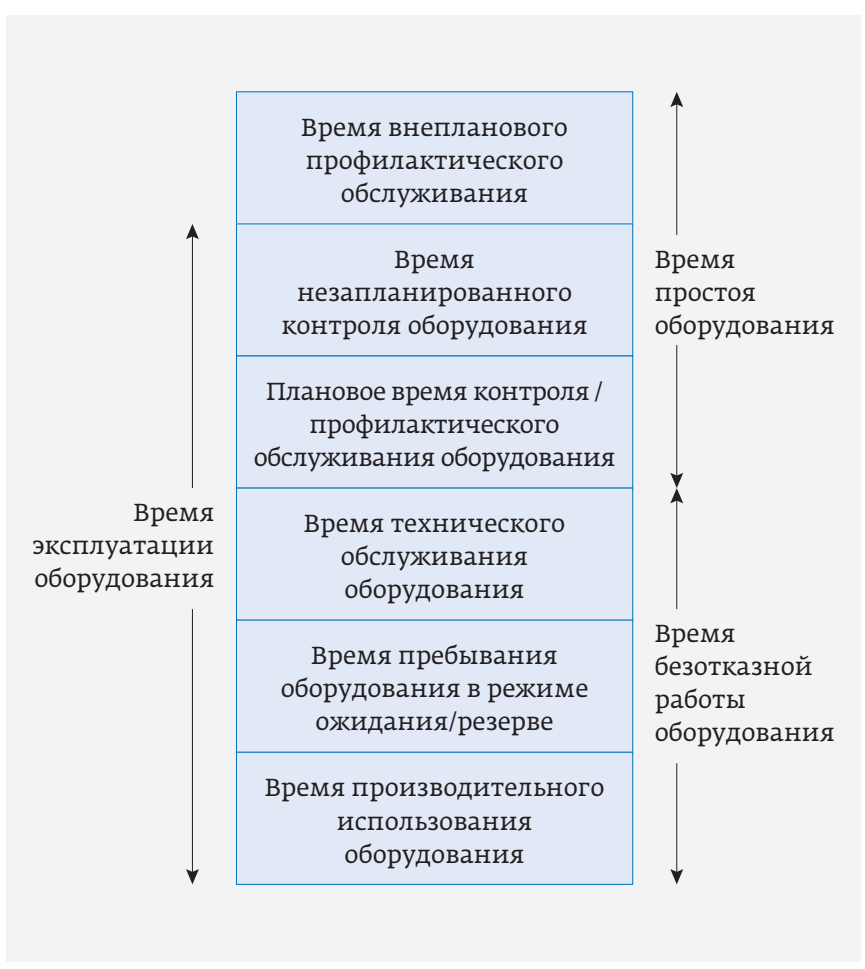

Рис. 4. Шесть основных состояний оборудования в соответствии со стандартом E10, разработанным SEMI

используемые методы двойного формирования рисунка, скорее всего, будут самосовмещающимися процессами с вырезными шаблонами, в отличие от LELE (litho-etch-lithoetch - двойной процесс "литография - травление» для каждого слоя) шаблонов расщепления. Подход EUV SADP с одним вырезным шаблоном, безусловно, вариант. Однако для эффективного разрешения пространственных ограничений может потребоваться более одного вырезного шаблона (скорее всего, избирательные/самосовмещающиеся вырезные шаблоны).

Исследователи Lam Research предлагают еще один взгляд на ситуацию: все зависит от предпочтений клиентов. Для некоторых вариантом может быть многократное формирование рисунка с самосовмещением. Это в основном касается тех разработчиков, которые освоили данный подход в рамках иммерсионного LELELE (litho-etch-lithoetch-litho-etch - тройной процесс «литография - травление» для каждого слоя) процесса, а теперь пытаются перенести этот опыт в сферу EUV-литографии. При этом возможно формирование 2D-конструкций с использованием двух прогонов полурисунков с увеличенным шагом, формируемых один в другой (что позволяет в результате добиться более плотного шага), за счет чего существенно повышается управляемость стохастическими явлениями.

IMEC разработал гибридный подход, сходный с методикой четырехкратного EUV-формирования рисунка. В этой методике осуществляются базовые EUV-этапы, затем применяется двухспейсерный процесс, а также процесс самосовмещения с двумя этапами литографии и двумя этапами травления (self-aligned litho-etch-litho-etch, SA / LELE). По существу, это гибридное - между SADP/ LELE и самосовмещающимися блоками - решение. SA/ LELE-процесс дает в целом бо́льшую гибкость одновременно с потенциальными возможностями контроля процесса. ІМЕС уже демонстрировал ряд процессов для формирования структур с шагом от 28 нм, теперь же начинаются работы по опробированию процесса, позволяющего добиться шага порядка 16 нм.

Еще один подход - сочетание EUV c DSA. Цель этого подхода прежде всего в экономии средств по сравнению с методикой двойного EUV-формирования рисунка. Данную методику предлагает корпорация Brewer Science (Рола, шт. Миссури). Данный подход позволяет достичь существенной экономии издержек за счет оптимизации времени использования дорогостоящих инструментальных средств EUV-литографии (издержки/час) при реализации стратегий многократного формирования рисунка. Кроме того, предлагаемая методика обладает потенциалом экономии издержек на различныхпромежуточныхшаблонах, закупаемых на основе требований к различным этапам EUV-экспонирования [4].

\section{ПЛАТФОРМЕННЫЙ ПОДХОД К МОНИТОРИНГУ РАБОТОСПОСОБНОСТИ ЕUV-СИСТЕМ}

По мере того, как EUV-литография осваивается в крупносерийном производстве, увеличивается потребность в оптимизации доступных систем, и одним из важных факторов является увязка наличного EUV-оборудования экспонирования с различными EUV-подсистемами. Подобные вопросы легче решаются на основе платформенного подхода. Одним из примеров являются работы фирмы Edwards. Для удовлетворения новых требований она создала на основе своей системы платформу EUV Zenith, позволяющую осуществлять мониторинг работоспособности системы с помощью дистанционного контроля параметров оборудования. Это обеспечивает быстрое время отклика за счет немедленного доступа корпоративных экспертов к проблемным зонам с целью осуществления прогнозных и корректирующих мероприятий по техническому обслуживанию для улучшения работы инструментальных средств и общей доступности.

Данная платформа действует в среде процессов и инструментальных средств EUV-литографии (ASML EUVL Process and Tool environment), созданной фирмой ASML, крупнейшим поставщиком полупроводникового производственного оборудования. Эта среда ориентирована на обеспечение доступности и производительность, где надежность и стабильность процесса занимают высокое место в рейтинге требований к производимой продукции и оборудованию. Определение этих требований с точки зрения sub fab oтражено в стандарте E10, разработанном SEMI и определяющим общие подходы, способ измерения эксплуатационных 
характеристик и производительности оборудования на основе шести его основных состояний (рис. 4) [6].

$$
\because * *
$$

Итак, несмотря на начало использования EUV-литографии в массовом производстве в качестве стандартной технологии, проблемы ее внедрения до конца не решены. Более того, по мере освоения появляются новые, ранее не предвиденные проблемы. EUV-литография в настоящий момент находится на ранней стадии применения, поэтому еще не может реализовать свой потенциал полностью. При этом возникает возможность, что на уровне 5-нм топологий могут понадобиться методики двойного EUV-формирования рисунка. Но методика двойного формирования может в процессе использования легко превращаться в методику тройного формирования и т.д. Другими словами, то, от чего пытались уйти за счет EUV в оптической литографии предшествующих поколений ИС, может вернуться уже на новом уровне. Возникает вопрос - а не зашла ли отрасль в тупик и не мешает ли накопленный парк оборудования, интересы его производителей дальнейшему развитию микроэлектроники? Может, выгоднее искать другие подходы развития, например использование голографических шаблонов и т.п... Разработка и применение последних по некоторым данным значительно проще и дешевле EUV-литографии [7].

\section{ЛИТЕРАТУРА}

1. EUV Lithography: Weighting The Options for Future Logic and Memory Applications // Semiconductor Digest. 2019. August/September. P. 22-24. http://online. publicationprinters.com/htm/5/reader/production/default. aspx?pubname=\&edid $=16 f 26302-22 c c-4140-84 f 3-f 0 f d b f 72 d c 4 e$

2. Derbyshire K. EUV Arrives, But More Issues Ahead // Semiconductor Engineering. 2019. March 21 ${ }^{\text {st }}$ https://semiengineering.com/euv-finally-arrives-now-what/

3. LaPedus M. EUV Mask Readiness Challenges//Semiconductor Engineering. 2019. March 21st.

https://semiengineering.com/euv-mask-readiness-challenges/

4. LaPedus M. Single Vs. Multi-Patterning EUV // Semiconductor Engineering. 2019. March 25th.

https://semiengineering.com/single-vs-multi-patterning-euv/

5. EUV Lithography: Sailing Among the Stochastic Cliffs // Semiconductor Digest. 2019. August/September. P. 25-28. http:// online.publicationprinters.com/htm/5/reader/production/default. aspx? pubname=\&edid=16f26302-22cc-4140-84f3-fofdbf72dc4e

6. Shannon Davis. Maximizing EUVL System Availability // Semiconductor Digest. 2018. July 11

https://www.semiconductor-digest.com/2019/07/11/ maximizing-euvl-system-availability/

7. Sub-wavelenght holographic lithography (SWHL): ideas, methods experimental verification. Nanotech SWHL GmbH. Dübendorf. Switzerland. 2019. June. www. nanotech-swhl.com

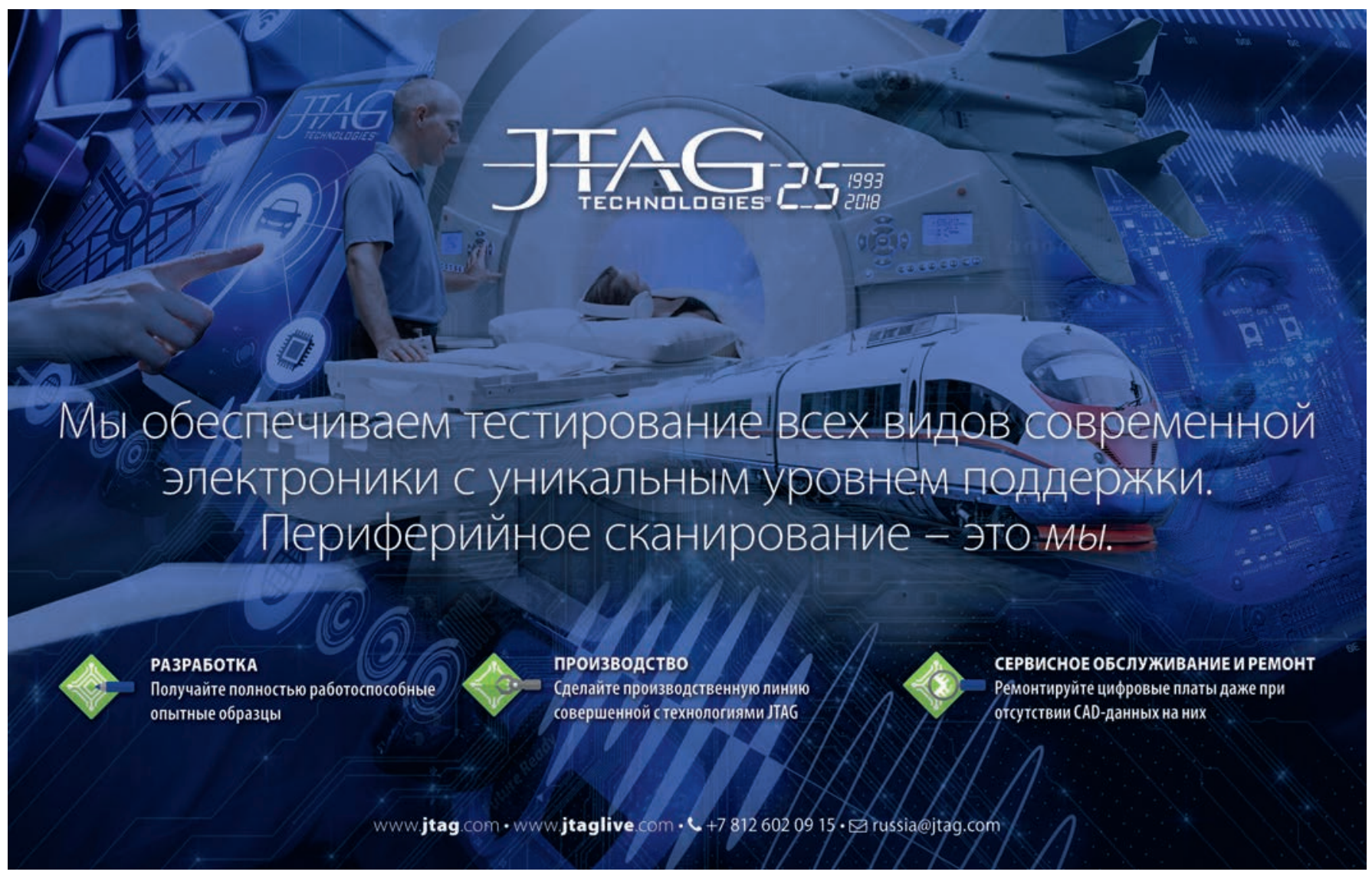

